\title{
Levante Selàf, Chanter plus haut. La chanson religieuse vernaculaire au Moyen Âge (essai de contextualisation)
}

\section{Paola Cifarelli}

\section{(2) OpenEdition}

1 Journals

\section{Édition électronique}

URL : http://journals.openedition.org/studifrancesi/6533

DOI : 10.4000/studifrancesi.6533

ISSN : 2421-5856

Éditeur

Rosenberg \& Sellier

\section{Édition imprimée}

Date de publication : 1 septembre 2010

Pagination : 333-334

ISSN : 0039-2944

\section{Référence électronique}

Paola Cifarelli, « Levante Selàf, Chanter plus haut. La chanson religieuse vernaculaire au Moyen Âge (essai de contextualisation)", Studi Francesi [En ligne], 161 (LIV | II) | 2010, mis en ligne le 30 novembre 2015, consulté le 08 janvier 2021. URL : http://journals.openedition.org/studifrancesi/6533 ; DOI : https:// doi.org/10.4000/studifrancesi.6533

Ce document a été généré automatiquement le 8 janvier 2021.

\section{(c)}

Studi Francesi è distribuita con Licenza Creative Commons Attribuzione - Non commerciale - Non opere derivate 4.0 Internazionale. 


\title{
Levante Selàf, Chanter plus haut. La chanson religieuse vernaculaire au Moyen Âge (essai de contextualisation)
}

\author{
Paola Cifarelli
}

\section{RÉFÉRENCE}

LEVANTE SELÀF, Chanter plus haut. La chanson religieuse vernaculaire au Moyen Âge (essai de contextualisation), Paris, Champion, 2008 («Nouvelle Bibliothèque du Moyen Âge» 87), pp. 650 .

1 La production lyrique vernaculaire d'argument religieux a certainement eu une fonction importante au sein de la dévotion populaire et n'a pas manqué de susciter un débat théologique animé. D'autre part, étant moulée sur les caractéristiques formelles de la poésie courtoise, elle n'était pas perçue comme un genre indépendant par les hommes du Moyen Âge: cela explique pourquoi les textes sont conservés pour la plupart dans des chansonniers composites qui mêlent des pièces d'argument sacré et des poèmes à contenu amoureux. La constitution du corpus qui fait l'objet de la présente analyse et qui comprend des textes français, galiciens et occitans, a donc présenté une série de difficultés qui sont exposées et discutées dans les deux chapitres liminaires.

2 Dans le premier, l'attention se focalise sur les textes bilingues qui constituent l'origine de la poésie religieuse vernaculaire, tels les épitres farcies et le drame liturgique connu sous le titre de Sponsus. Par contre, le chapitre 2 expose les principes méthodologiques utilisés pour la détermination du corpus: analyse critique des critères d'inclusion et de classifications des textes dans les répertoires, discussion des définitions proposées pour le dit, problèmes concernant la structure métrique et strophique, liés à la conformité au style gonique et au caractère chanté ou non chanté des poèmes. Enfin, la reconstruction du contexte manuscrit, littéraire et historique des chansons, 
permettrait de pallier les insuffisances d'une méthode fondée sur l'analyse formelle et fournir les moyens pour définir et caractériser les textes.

3 Pour ce qui est de la production en ancien français, L.S. parvient à sélectionner 297 textes et se propose d'en cerner les spécificités stylistiques, d'en retrouver les points communs et de définir la place occupée par ce qu'il considère comme un véritable genre dans l'ensemble de la «poétique médiévale» (p. 59).

4 La première composition qui fait l'objet d'une analyse détaillée est représentée par les Vers de la mort d'Hélinand de Froidmont. Le douzain d'octosyllabes utilisé dans cet ouvrage deviendra extrêmement populaire dans le contexte pieux et sera repris par plusieurs auteurs, à tel point que, selon l'A., le poème 'en strophe d'Hélinand' constituerait à lui seul un véritable genre; en effet, certaines particularités métriques (hétérogonie, proportion des rimes féminines créant un effet incantatoire, début de vers analogique) deviendront caractéristiques des poèmes religieux qui mettent l'accent sur l'aspect moral, ainsi que des complaintes (religieuses ou non), équivalent latin du planctus.

5 Une première subdivision du corpus s'esquisse donc; l'observation des substantifs utilisés pour désigner les poèmes du corpus est le critère utilisé dans le chapitre 3 pour essayer de retrouver d'autres sous-ensembles possibles; les dénominations employées par les philologues sont comparées à celles que l'on retrouve dans les traités de poétique ou par les copistes des chansonniers, et enfin à celles, nombreuses et variées, qui apparaissent à l'intérieur des textes eux-mêmes. Parmi ces derniers, c'est le terme serventois qui fait l'objet d'une analyse particulièrement fine, permettant à l'auteur de s'interroger sur l'utilisation de ce mot pour désigner tout poème commandé ou offert. Plus généralement, l'A. parvient à la conclusion qu'aucune terminologie spécifique n'est utilisée au Moyen Âge pour désigner les poèmes pieux, conformément au principe selon lequel la perception de l'appartenance de ceux-ci à un genre à part appartient à la mentalité moderne. En même temps, ce chapitre propose une réflexion sur la notion de genre, ou plutôt de «système de genres» (p. 163).

6 Alors que les chapitres 4 et 5 sont consacrés à la poésie lyrique occitane et galicienne, le chapitre 6 est focalisé sur les problèmes liés à l'imitation, surtout pour ce qui concerne la mélodie et la formule métrique: en effet, celle-ci est souvent reprise d'une tradition profane plus ancienne, selon la technique bien connue de la contrafacture. C'est donc à cette notion, et plus généralement au concept de 'nouveauté' qu'est consacrée cette section de la présente étude; l'A. analyse la production de Gautier de Coinci et de Jacques de Cambrai, puis les textes contenus dans plusieurs chansonniers bien connus ( $\mathrm{X}, \mathrm{C}$ et $\mathrm{V}$ ) et enfin les Cantiques Salemon, traduction commentée du Cantique des Cantiques. L'hypothèse de travail, confirmée par l'étude des textes, est que la contrafacture pourrait parfois être utilisée dans un but polémique vis-à-vis de l'amour profane, auquel les chansons religieuses opposent un sentiment plus haut: le concept d'imitation devrait donc être considéré sous un angle plus vaste et dans toute sa complexité, plutôt que comme la conséquence d'un manque de qualité artistique.

7 Après avoir abordé la question des origines de la chanson pieuse dans le chapitre 7, l'A. examine les motifs textuels et métriques de son corpus en ancien français, toujours dans le but d'identifier des tendances spécifiques et d'isoler des sous-genres. Une approche plus sociologique est adoptée pour la section suivante, qui aborde la question du public des chansons pieuses et de la circulation de certains textes dans des milieux déterminés (courtois, bourgeois, religieux). C'est ensuite la typologie des manuscrits 
conservant des chansons pieuses qui est examinée dans le chapitre 10; à côté des chansonniers proprement dits, l'analyse s'étend aux recueils entièrement formés de poèmes pieux essentiellement mariaux, tels les Miracles de Nostre Dame et le Rosarius, puis aux mystères et autres drames liturgiques, et enfin au Roman dou Lis, exemple de texte narratif d'inspiration mariale intercalé par plusieurs chansons. Une section à part est réservée à la vulgarisation du Ludus super Anticlaudianus d'Adam de la Bassée et à ses insertions lyriques.

8 Le dernier chapitre a comme objet les compositions pieuses accompagnées de commentaires, qui permettent de nous renseigner sur l'interprétation que recevaient ces textes au Moyen Âge. Des considérations sur les possibilités de réalisation d'un répertoire métrique général des strophes à l'époque médiévale achèvent cette étude, complétée par deux Appendices (l'édition des chansons contenues dans le ms 43 de la Bibliothèque de la Faculté de Médecine de l'Université de Montpellier et la liste des chansons religieuses occitanes présentées dans le texte), par une bibliographie très riche et par les index. 